\title{
A Novel Weighted Median Switching Filter for Denoising Corrupted Images
}

\author{
Saurabh Agrawal \\ PhD Scholar \\ MANIT, EC Dept. \\ Link Road No.3, Vaishali Nagar \\ Bhopal, MP - 462051
}

\author{
J. S Yadav, PhD. \\ Associate Professor \\ MANIT, EC Dept., \\ Link Road No.3, Vaishali Nagar \\ Bhopal, MP - 462051
}

\author{
Ravindranath C. C, PhD. \\ Professor, Head of Department \\ GGITM, EE Dept. \\ Raisen Road, Anand Nagar \\ Bhopal, MP - 462021
}

\begin{abstract}
This paper presents a new weighted switching median filter is proposed to restore images corrupted by noise. The neighborhood of a pixel is classified into low corrupted, medium corrupted and highly corrupted regions. Based on this classification the weights associated with the median filter employed for denosing is determined. In addition, switching mechanism allows the use of different weights for different regions based on localized statistics. The proposed algorithm can be applicable to digital images with most of the fine details of the image are mostly preserved. The simulation analysis would shows that the proposed method is good for noise reduction and image restoration.
\end{abstract}

\section{General Terms}

Denoising, Security, Median Filter, Image Processing

\section{Keywords}

Data modeling, denoising, noise region detection, weighted median filter, non-linear filter, threshold segmentation

\section{INTRODUCTION}

Denoising is a process of suppressing noise while preserving the integrity (such as edges and texture) of the image. In the current digital era, the signals getting corrupted during the transmission had become quite common. This may be caused due to noisy transmission lines, instabilities in circuitry, and/or environmental or weather fluctuations. Further, during the course of acquisition these signals could also be corrupted due improper handling of the acquisition tools or the manner they were acquired. Noise affects the perceptual quality of the image, decreasing not only the appreciation of the image but also the performance of the task for which the image was intended.

In the field of communication, digital images are valid information signals employed during transmission. Therefore, image denosing used prior subsequent processing steps such as image analysis and compression is an essential part of any image processing system. Henceforth, noise removal algorithms are essential for signal restoration to precede reliable digital signals/ images through varied image processing applications. Denosing algorithms can successfully address this issue while preserving the detail information of the image that could be subsequently used for edge detection, image segmentation or object recognition based image processing operations.

There are several approaches proposed that could remove the noise from the corrupted images. They can be classified into two classes, namely, spatial methods and frequency methods. Most of the spatial smoothing processes as the mean and the median filters, which are widely used [1-4], generally tend to remove noise without explicitly identifying it. It is, though, possible to filter selectively the noise signal by comparing the gradient grey level in a neighborhood to a fixed threshold [2]. The frequency smoothing methods [1] remove the noise by designing a frequency filter and by adapting a cutoff frequency when the noise components are decorrelated from the useful signal in the frequency domain. Unfortunately, these methods are time consuming and depend on the cut-off frequency and the filter function behavior. Furthermore, they may produce artificial frequencies in the processed image. In this paper, we concentrate on Non-Linear approaches are direct evaluation methods that are more manual in nature based on statistics in time domain.

Much of the potential research is being concentrated in developing non-linear approaches as compared to linear approaches [3-4]. The linear approaches could not distinguish noise from underlying signal that causes needless distortion to the signals. Further, linear approaches show poor performances against impulse noise. For example, denoising algorithms based on linear operations smoothened image details while removing noise and so non-linear operators emerged successful to deal the non-linear characteristics of impulses.

In this paper, we introduce a novel weighted median switching filter for denosing corrupted images. The proposed system uses localized statistics to classify the corrupted image pixels into low, medium and high classes. Then each class is filtered based on weighted median filter whose weights are automatically determined based image statistics. The switching action takes place based on the noise detection. Further, the proposed system offers better noise detection by minimizing the false detection of noise pixels. It also shows excellent simulation results against various noise and level of corruption in consideration.

The following paper has been organized as follows. In this correspondence, Section 2 describes about various existing denosing techniques and the basic architecture of the switching filter. Section 3 presents the new framework for classifying each image pixels into a noisy or non-noisy pixels based on localized image statistics. The proposed system and its algorithm are explained in section 4 with detail illustrations. Section 5 deals with the experiments results and the security analysis of the algorithm. Section 6 concludes the paper.

\section{BACKGROUND}

In this section, we introduce the basic architecture of the switching filter and existing denosing techniques. There are various types of noise. However, they fall into two major classes: additive and multiplicative noise [5]. An example of 
multiplicative noise is variable illumination. This is perhaps the most common type of noise in images.

Additive noise is often assumed to be Impulse noise or Gaussian noise which is perhaps the most common type of noise that occurs during digital media transmission. Impulse noise alters at random the value of some pixels. In a binary image this means that some black pixels become white and some white pixels become black. This is why this noise is also called salt and pepper noise [6]. Additive zero-mean Gaussian noise means that a value drawn from a zero-mean Gaussian probability density function is added to the true value of every pixel.

In general, we consider two aspects in designing a switching filter i.e. noise detector and noise estimator (filter). The efficiency and robustness of the switching filter depends on how well the noise detector can differentiate between various types of noises within the given digital media. In case of estimator[7-11], we are employing a rank order filter as a denoising filter. A filter whose the output value depends on the ranking of the pixels according to their grey values inside the filter window is called rank order filter. The most common rank order filter is the median filter. The basic architecture of the switching filter is illustrated in the figure 1.

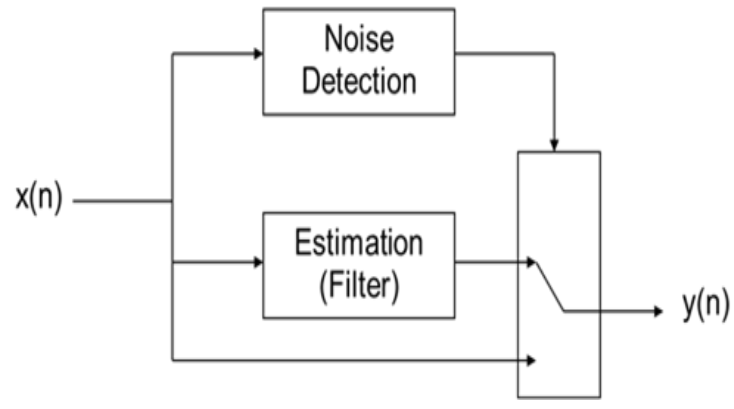

Fig. 1Basic architecture of the switching filter

\subsection{Noise Detection}

The role of noise detection is vital as it can considerable improve the efficiency of the denosing system. Further, it can also reduce over-filtering and preserve image details and structure to maximum extent. Henceforth, these algorithms have received considerable attention in image processing research over the last two decades [8-10]. A. Beghdahi and A. Khellaf [10] proposed entropy based noise detection algorithm for impulse noise, which was very effective and robust but showed poor response for Gaussian noise and mixture of both.

Abreu, E. and S.K. Mitra [11] used a variation measure based on the standard deviation of the localized image statistics. Further, to improve its robustness they created a training sets based on these measure. This framework could successfully classify impulse noise regions. Unfortunately, it did not offer promising analysis in case of the Gaussian noise.

\subsection{Noise Estimator}

One important characteristic of the median based denoising consists of the fact that it does not produce values for a pixel that do not exist in the original image. The median is the grey level value which divides a distribution in two equally numbered populations. For example, if we use a $3 \times 3$ window, we have 9 grey level values which we order in an increasing sequence. Then the median value is the fifth value.
This method has the effect of forcing points with distinct intensities to be more like their neighbors, thus eliminating intensity spikes which appear isolated. Consider the pixels $X=\left\{x_{1}, x_{2}, x_{3}, \ldots x_{n}\right\}$ then the median of these pixels is calculated by sort these pixels into increasing order. Then the median of the sequence is given by

$$
\operatorname{Median}[X]=\left\{\begin{array}{c}
x_{(n+1) / 2} \text { if } n \text { is odd } \\
\frac{\left(x_{n / 2}+x_{(n+2) / 2}\right)}{2} \text { if } n \text { is even }
\end{array}\right.
$$

Assuming that $x_{1},<x_{2}<\ldots<x_{n-1}<x_{n}$. Unfortunately, using the median framework and the universal denosing method throughout the image would result in following shortcoming:

- This system would denoise all the pixels of the image in consideration without analyzing if the pixel is corrupted or not.

- The system might contribute to the needless degradation of image details and fine features.

- In case of image corrupted with Gaussian noise, it offers poor filtration.

Henceforth, we need to design a denoising method that could address each noise structure with a specific framework for filtering.

\section{PIXEL SELECTION}

In this section, we introduce a method based on the localized statistics for analyzing corrupted pixels to non-corrupted pixels within an image. We have designed variations measures based on the localized statistical calculations that are dependent on median based statistics. Thus, algorithms designed based on these measures offer increased accuracy in the separation and identification of different types of noise.

$$
\rho=\frac{1}{N} \sum_{n=0}^{N}\left(x_{n}-\operatorname{Median}\left\{x_{n} \| n \in[1, \ldots, N]\right\}\right)^{2}
$$

Where,

" $\rho$ "indicates the variation designation

"N" indicates the number of pixels in the window

" $x_{n}$ " indicates the iterative pixel of the window

By using, local variation based median statistics of neighboring pixels gives stronger foundation about the pixel in consideration. In addition, it offers room for error by incorporating method to remove contribution of corrupted pixels to local statistics. Directional variation measure allows for mapping of fluctuations along different axis as presented in the figure 2. It is a well-known fact that directional variation measure based median analysis provides a through classification based on orientation with respect to center pixel. The ability to selectively classify corrupted to non-corrupted pixel allows the reduction in false detection of pixels.

This system offers the ability to reconstruct images corrupted with extreme levels of noise. Moreover, automatic threshold calculation presents as additional advantage in comparison with the existing systems. The general classification of the pixels in the image based on the proposed framework is illustrated in the figure 3 . 

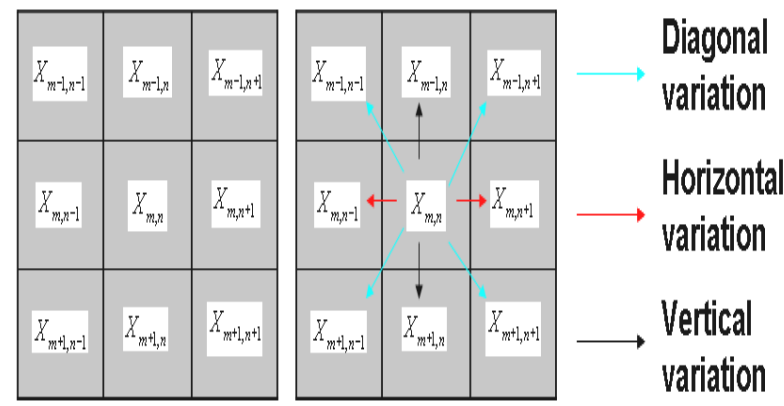

Fig. 2 Different variations in a $3 \times 3$ block

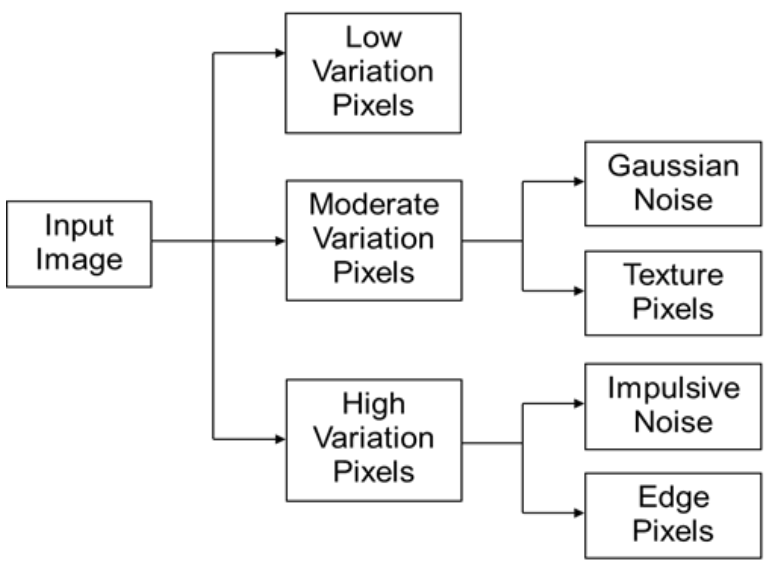

Fig. 3 The general classification of the pixels

The initial threshold " $\rho_{s}$ " is derived by constructing histograms of all the variations of the pixels within the image. This threshold differentiates between the smooth regions and non-smooth regions (homogeneous regions). Thus, over filtering issues are readily addressed using this classification. The effect of this measure has been illustrated in the figure 4 , where figure 4(a) is the original image and the figure 4(b) is the homogenous area and the blacken spots are the estimated smooth regions as per the proposed variation.

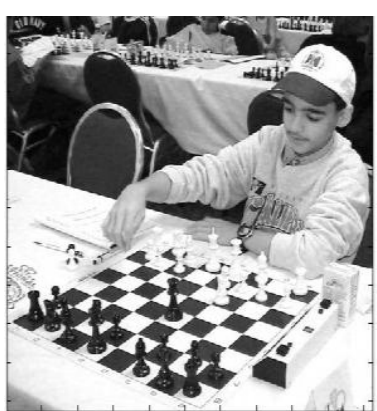

a) b)

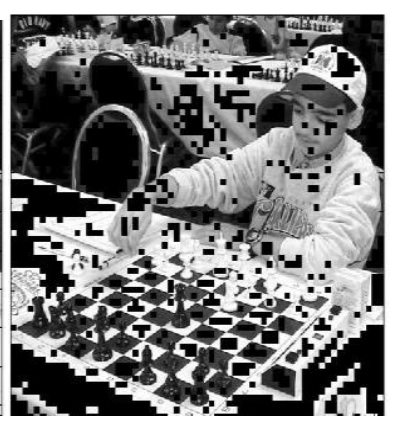

Fig. 4 a) The original image \& b) Homogeneous areas within the image

The next variation measure is employed to differentiate between moderate to high variation pixels based on the threshold measure " $\rho_{g}$ ". The expression associated with this threshold measure is given as

$$
\rho_{g}=\frac{1}{N * M} \sum_{i=1}^{N} \sum_{j=1}^{M} \rho(i, j)
$$

Where,
" $\rho_{g}$ " indicates the second threshold measure

"N" \& "M" indicates the size of the image

" $\rho$ " indicates the variation measure of each pixel

The basic classification of the pixels is into three groups based on the threshold measure i.e. as follows

$$
\begin{array}{llc}
\text { 1. } & \text { Low Variation Pixels } & \rho<\rho_{s} \\
\text { 2. } & \text { Moderate Variation Pixels } & \rho_{s}<\rho<\rho_{g} \\
\text { 3. } & \text { High Variation Pixels } & \rho_{g}<\rho
\end{array}
$$

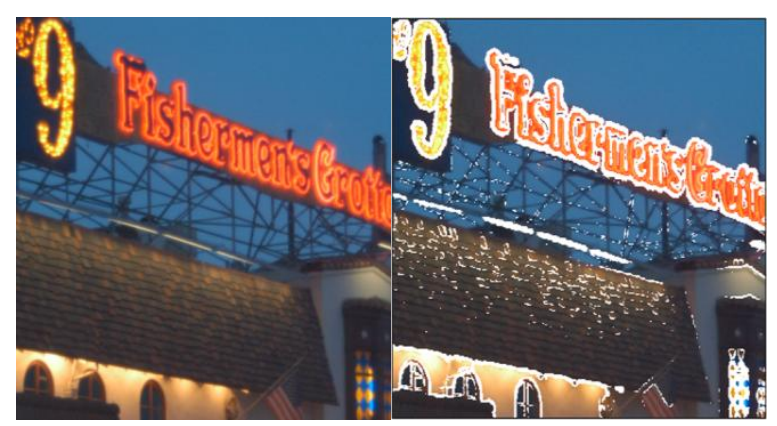

a)

b)

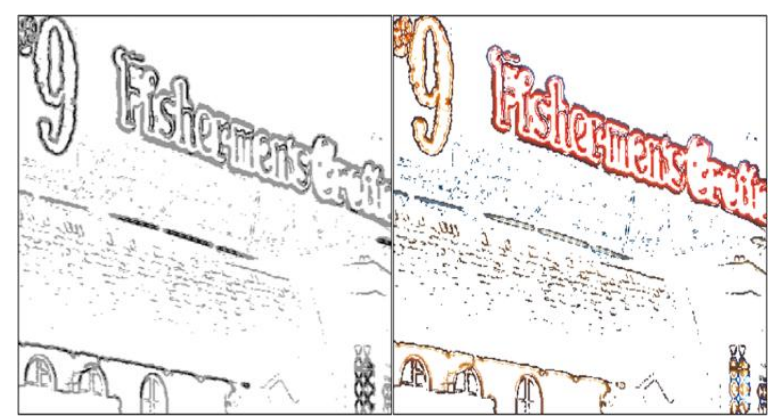

c)

d)

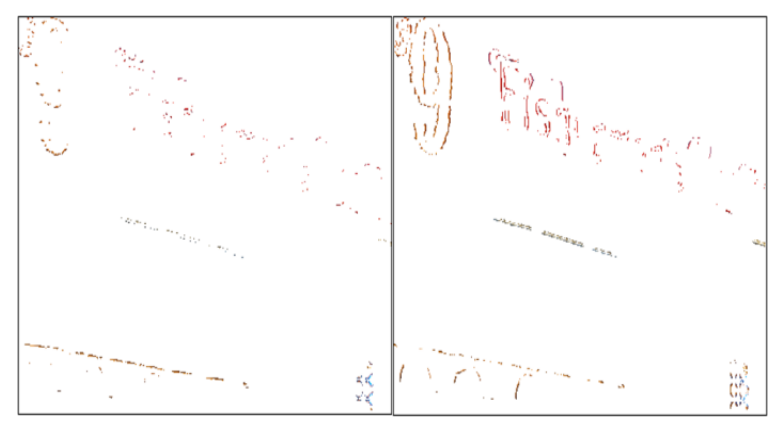

e)

f)

Fig. 5 a) The original image b) possible low variation pixels c) texture pixels d) edge pixels e) Gaussian Noise pixels and f) Impulse noise pixels

The High variation pixels comprise of the edge pixels and impulse noise pixels which can be classified based on the variations measures (i.e. horizontal, vertical and diagonal) discussed in the earlier section. In this paper, we limit our focus to horizontal and vertical directional measures. These two sets are calculated for every high variation pixel in the image and the expression is evaluated as follows

$$
\alpha=\frac{\operatorname{Max}\left({p_{h}}^{2}\right)}{\operatorname{Min}\left({p_{v}}^{2}\right)}
$$

Where, 
" $\alpha$ " indicates the directional variation measure ratio and " $\rho_{h}$ " $\&$ " $\rho_{v}$ " indicates the two sets of the directional measures for high variation pixels. The same propose is carried out for the classification of the Gaussian noise pixels to the texture pixels. The various classes of pixels in a given image are presented below in figure 5 .

\section{PROPOSED SYSTEM}

In this section, we introduce a new weighted switching filter for denoising corrupted digital images. The basic block diagram of the proposed system is illustrated in the figure 6 .

Input image: The input image may be of any image format using the 8-bit, power of two's representation. The proposed algorithm may also be applied on 24-bit, RGB images treating individual layers as an individual 8-bit image.

Threshold Calculation. The variation measure for each pixel is calculated " $\rho$ ". Then the corresponding thresholds are calculated as per the expression presented in the above section. Thus, we have the following threshold values " $\rho_{s}, \rho_{g}$ and $\alpha$ ". " $\alpha$ " is calculated only for the pixels whose variation measure " $\rho$ " is greater than " $\rho_{g}$ ".

System 1: Pixel Classification. This block is the first stage of the proposed denoising system. Based on the thresholds every pixel within the image is classified into the following groups as presented below:

Smooth \& Texture pixel

$$
\begin{gathered}
\rho<\rho_{s} \\
\rho_{s}<\rho<\rho_{g} \\
\rho_{g}<\rho \& \alpha<\alpha_{n} \\
\rho_{g}<\rho \& \alpha \geq \alpha_{n}
\end{gathered}
$$$$
\text { Gaussian Noise pixel }
$$$$
\text { Impulse Noise pixel }
$$

Edge pixel

Where, " $\alpha_{n}$ " is the final threshold that would classify between impulse noise and edge pixels.

System 2: Pixel Estimator. This block is the second stage of the proposed denoising system where actual filtering of the noise takes place. Any pixel of the corrupted image identified as a smooth, texture, edge pixel is un-altered and passed directly as the denoised output. But in case of Impulse and Gaussian noise pixel weighted median filter is applied.

Weights associated with the median filter are altered based on the pixel in consideration. If the pixel in consideration is an impulse noise pixel then we use regular median filter architecture. But if the pixel in consideration is a Gaussian
Noise pixel then the weights associated with the median filter are altered and based on the LMW, MW, HMW and the corresponding output is determine as the denoised output.

LMW, MW, and HMW (indicates the low median weight, median weight, high median weights respectively) that are determined based on localized statistics. For a given localized window, we determine three medians based on distribution with in the region of interest. Pixels whose values are below median value are called low median pixels and pixels whose values are above median are called high median pixels.

For each class, the median is determined and the average of two classes along with median value of the localized window determines the filter value output on the center pixel. Consider the pixels $X=\left\{x_{1}, x_{2}, x_{3}, \ldots . x_{n}\right\}$ then the median of these pixels is given bymed $[X]$. Then the expressions of LMW, MW, and HMW are as follows

$$
\begin{array}{r}
L M W=\operatorname{Median}\left\{x_{i} \leq \operatorname{med}[X]\right\} \quad i \in[1,2, \ldots n] \\
M W=\operatorname{med}[X] \\
H M W=\operatorname{Median}\left\{x_{i} \geq \operatorname{med}[X]\right\} \quad i \in[1,2, \ldots n]
\end{array}
$$

The filter output of the proposed filtering is obtained based on the equation given below

$$
\hat{X}=\frac{\beta_{1} * L M W+\beta_{2} * M W+\beta_{3} * H M W}{\beta_{1}+\beta_{2}+\beta_{3}}
$$

Where, $\beta_{1}, \beta_{2}, \beta_{3}$ are the weights associated with the each of the localized statistics determined earlier, further for simulations purpose the values of the each weight have been fixed as $0.85,1.2$, and 0.8 . Currently, research is being carried out to determine the impact of each weight on the statistic with respect to the denoising of the corrupted region.

\section{Output:Denoised image.}

The proposed systemis quite easy to use and simply to alter based on the requirement, as all necessary parameters are dictated based on the statistics of the corrupted image. Further, the base of the system is threshold calculation that classifies pixels into various classes which could be used in various security applications as well. Further, the filtering algorithms are independent with reference to proposed system hence forth they could be altered to enhance the performance of the proposed system.

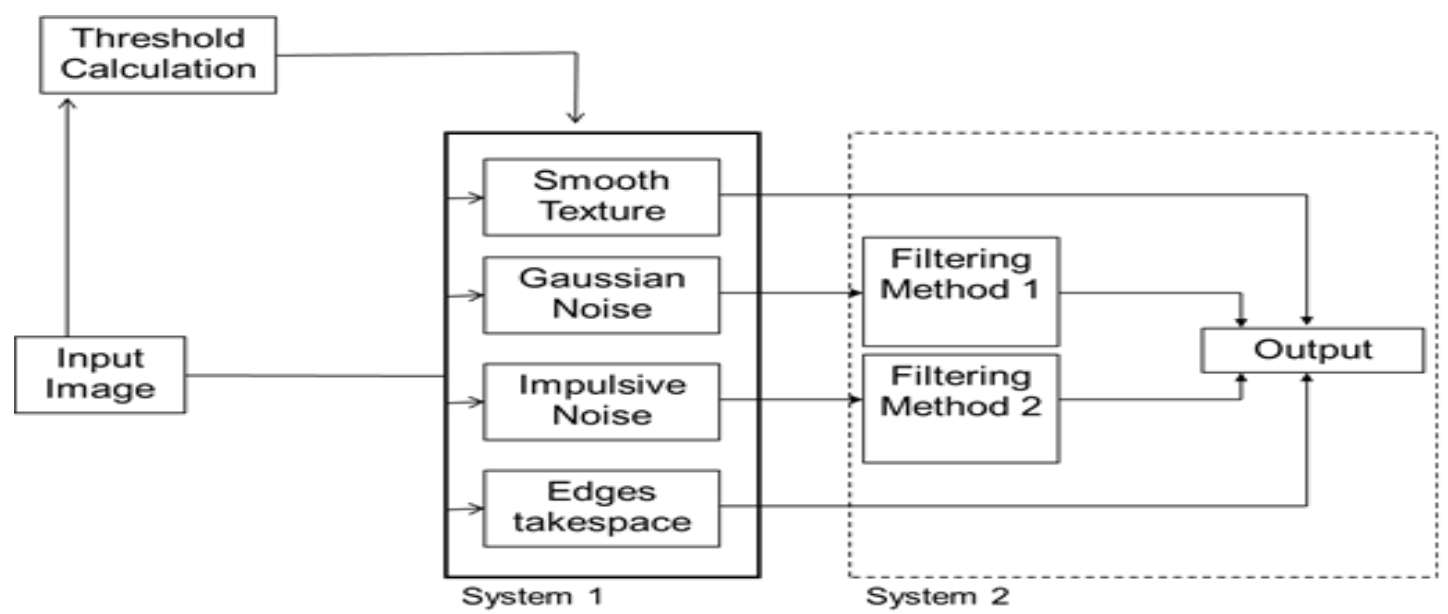

Fig. 6 The general structure of the weighted switching median filter for denosing corrupted images 


\section{COMPUTER SIMULATIONS}

In this section, the simulations results of proposed security system are presented. Computer simulations were simulated using MATLAB software package. Analysis was carried out using 100 digital images of varying formats, sizes, texture and contour (simulations are presented for few images). These images were taken using 2 digital cameras Nikon D100 and Canon EOS Digital Rebel and modified in Photoshop to attain a smooth histogram.

\subsection{Statistical Analysis}

Initial investigation was based on the statistical analysis between the corrupted image and the corresponding denoised image. A good measure would be able to identify the value regarding, how much an image has changed from applying proposed denoising technique. This evaluation measure is based on the calculation of the MSE value as presented below

$$
M S E=\frac{1}{N * M} \sum_{i=1}^{N} \sum_{j=1}^{M}(x(i, j)-y(i, j))^{2}
$$

Where, " $x$ " indicates the original image and " $y$ " indicates the denoised image of size "N" and "M".

Table 1. MSE of the corrupted and filtered images with reference to original uncorrupted image

\begin{tabular}{|c|c|c|c|c|}
\hline Images & Lena & Sarkis & Fisherman & Rose \\
\hline $\begin{array}{c}10 \% \\
\text { Impulse }\end{array}$ & 1147 & 1043 & 1223 & 968 \\
\hline $\begin{array}{c}10 \% \\
\text { Impulse } \\
\text { (filtering) }\end{array}$ & 102 & 212 & 133 & 87 \\
\hline $\begin{array}{c}0.1 \text { var } \\
\text { Gaussian }\end{array}$ & 690 & 1025 & 865 & 632 \\
\hline $\begin{array}{c}0.1 \text { var } \\
\text { Gaussian } \\
\text { (filtering) }\end{array}$ & 44 & 93 & 105 & 45 \\
\hline $\begin{array}{c}10 \%+0.1 \text { var } \\
\text { Both }\end{array}$ & 1623 & 1945 & 2013 & 1476 \\
\hline $\begin{array}{c}10 \%+0.1 \text { var } \\
\text { Both } \\
\text { (filtering) }\end{array}$ & 69 & 253 & 162 & 110 \\
\hline $\begin{array}{c}\text { Clean Image } \\
\text { (filtering) }\end{array}$ & 31 & 62 & 59 & 24 \\
\hline
\end{tabular}

The mean square error of each image that was corrupted and denoised with reference to the original uncorrupted image is presented in the Table 1. Further, for better analysis, we the proposed denosing on the original uncorrupted image to investigate its affect and the system was denosing information this was due to the natural noise that normally present in an image is due to the following reasons.

1) due to light condition during which the image is taken,

2) due to the hard ware employed to take the image and

3) due to the handling of device while taking the image.

It is evident from the table 1,the proposed system offers a robust denosing mechanism for the corrupted image.

\subsection{Visual Analysis}

In order to test the robustness of the proposed system after denoising, we performed human visual system (HVS) comparison test. In this test, we selected a group of 25 volunteers from the electronics and communication department at "Maulana Azad National Institute of Technology, Bhopal" and asked them to rate (0 to 10) the each Denoised Image they saw is close to the original image with reference to corrupted image. For this case, we used varying capacities of noise [such as $10 \%$ impulse, $10 \%$ Gaussian and 10\% each Impulse and Gaussian].

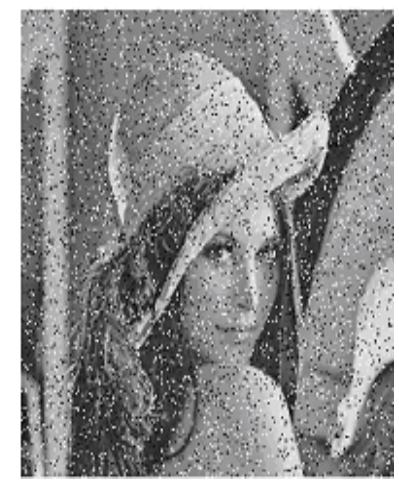

a)

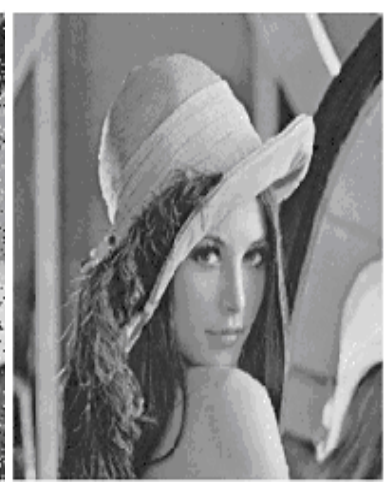

b)
Fig. 7 a) The corrupted image with $10 \%$ impulse noise b) denoised image

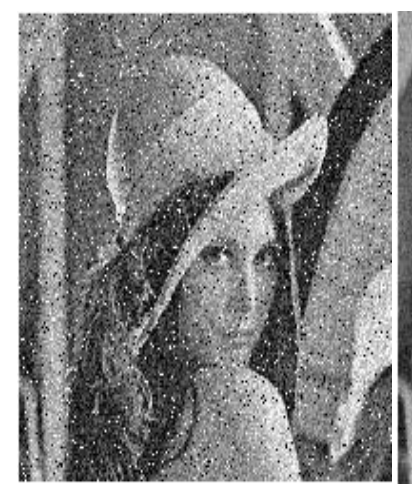

a)

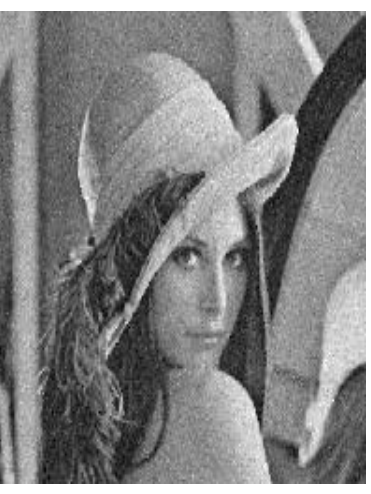

b)
Fig. 8 a) The corrupted image with 0.1 variance Gaussian noise b) denoised image

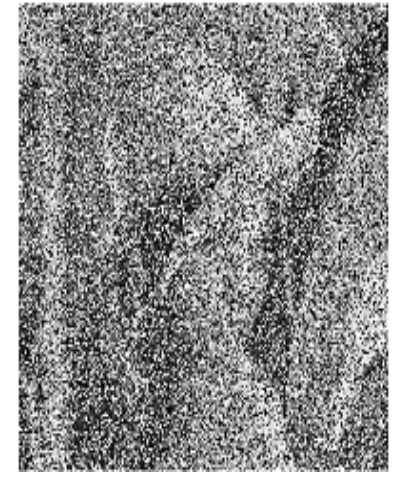

a)

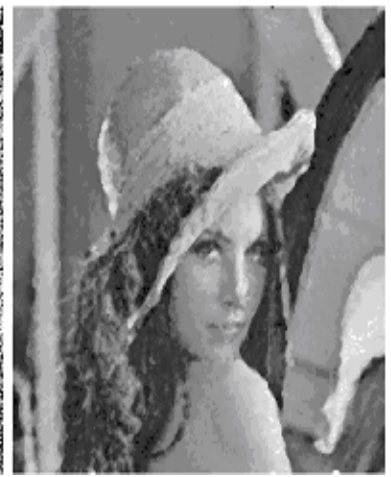

b)
Fig. 9 a) The corrupted image with $10 \%$ impulse noise and 0.1 variance Gaussian noise b) denoised image

The figure 7 shows the corrupted image with $10 \%$ impulse noise and the corresponding denoised image. The figure 8 shows the corrupted image with 0.1 variance Gaussian noise and the corresponding denoised image. The figure 9 shows the corrupted image with $10 \%$ impulse noise over which 0.1 
variance Gaussian noise is added and the corresponding denoised image.Maximum volunteers voted that the maximum features of the corrupted image were preserved and we have received a rating of $89 \%$ (on average).

\section{CONCLUSION}

We have introduced a new weighted switching median filter for restoring images that have been corrupted by noise. We have also successfully classified all the pixels of the image into Smooth-texture, Gaussian noise, Impulse noise and edge pixels with a minimal false error. We have also successfully addressed the issues regarding the weights associated with the median filter employed for denosing. In addition, switching mechanism allowed the use of different weights for different regions based on localized statistics. Further, the proposed algorithm have successfully applied to digital images with most of the fine details of the image are preserved. The simulation analysis also proved that proposed method is good for noise reduction and image restoration even if the images have high amount distortion.

\section{REFERENCES}

[1] Astola, Jaako and Pauli Kuosmanen, "Fundamentals of non-linear digital filtering", CRC Press. Boca Raton, New York, 1997

[2] R. C. Gonzales and R. E. Woods, "Digital Image Processing”, Addison-Wesley, New York, 1992.

[3] S. Jayaraman, S. Esakkirajan and T. Veerakumar, "Digital Image Processing", Tata McGraw Hill, New Delhi, 2009.

[4] Ashima mittal, Akash Taval "A Deatailed Comparison of Switching Median Filters" International Journal of
Information Technology and Knowledge Management, July-Dec 2012, Vol.5, No.2, pp 439-442

[5] L. Yin, R. Yang, and M. Gabbouj, "Weighted median filters: A tutorial," IEEE Transactions on Circuits Systems II: Analog Digital Signal Processing, vol. 43, pp. 157-192, March 1996

[6] T. Sun, M. Gabbouj, and Y. Neuvo, "Center weighted median filter: some properties and their applications in image processing," Signal Processing, vol. 35, pp. 213 229, Feb. 1994.

[7] Beghdadi, Azeddine and Amar Khellaf, "A NoiseFiltering Method Using a Local Information Measure", IEEE Transactions on Image Processing, Vol.6, No. 6, June 1997.

[8] Abreu, E. and S.K. Mitra, "Signal Dependent Rank Ordered Mean (SD-ROM) Filter - A New Approach for Removal of Impulses From Highly Corrupted Images", IEEE Transactions on Image Processing, May 1995.

[9] S. Ko and Y. Lee. "Center Weighted Median Filters and Their Applications to Image Enhancement," IEEE Transactions on Circuits and Systems, Vol. 38, No. 9, Sept 1991.

[10] Shmulevich and G. Arce. "Spectral Design of Weighted Median Filters Admitting Negative Weights," IEEE Signal Processing Letters, Vol. 8, No. 12, Dec 2001.

[11] Z. Wang and D. Zhang. "Progressive Switching Median Filter for the Removal of Impulse Noise from Highly Corrupted Images," IEEE Transactions on Circuits and Systems II: Analog and Digital Signal Processing, Vol. 46, No. 1, Jan 1999. 